\title{
Dal rito dell’incubazione alla psicoterapia
}

\section{Una prospettiva transculturale}

\author{
Tania Re \\ Psychotherapie-Wissenschaft 9 (1) 62-67 2019 \\ www.psychotherapie-wissenschaft.info \\ CC BY-NC-ND \\ https://doi.org/10.30820/1664-9583-2019-1-62
}

\begin{abstract}
Riassunto: In origine, in Occidente la medicina che prevedeva la cura del corpo, della mente e dello spirito era un'arte divinatoria. Nel corso del tempo il termine «divinazione» ha perso il suo significato originale nel corso dei secoli, rispetto ai tempi antichi e, in particolare, al mondo arcaico greco, in cui ha avuto un enorme valore. L'arte di Asclepio, è, come quella del padre Apollo, arte divina che presuppone un rito di guarigione. A questo proposito, possiamo ricordare che in ambito terapeutico le ricerche storico-antropologiche registrano in molte culture di tipo sciamanico (Sud America, Siberia, India) un utilizzo molto antico di "piante maestre", ovvero piante che, grazie alle loro proprietà, permettono di raggiungere "Stati non ordinari» di coscienza. Tali stati di coscienza possono sorgere attraverso diverse pratiche, come la meditazione, la respirazione olotropica, lo yoga, la deprivazione sensoriale, o addirittura a livello spontaneo come nel caso dei mistici cristiani. L'esperienza contemporanea riportata dai ricercatori ci sta offendo dati molto incoraggianti sui benefici di questo tipo di esperienze. La ricerca sviluppata negli USA e in Europa in Spagna e Svizzera, tende a seguire protocolli di ricerca, in cui giocano un ruolo fondamentale il Set (lo stato attuale ma anche la storia clinica e biografica del paziente), il Setting (l'ambiente in cui avviene l'esperienza) e la sostanza (tipologia, dosaggio, qualità e quantità). Le sfide per il futuro in ambito psicoterapeutico sull'utilizzo terapeutico delle sostanze psicoattive sono ancora molte, una possibilità concreta potrebbe essere quella di rivitalizzare l'utilizzo rituale delle sostanze unendo passato e presente, antiche conoscenze e moderna scienza a servizio della cura.
\end{abstract}

Parole chiave: Asclepio, Epidauro, Medicina sapienziale, Medicina templare, Santuario dell'incubazione, Piante maestre, Sciamanesimo

\section{Le origini: la medicina negli Asclepieion}

In origine, in Occidente la medicina che prevedeva la cura del corpo, della mente e dello spirito era un'arte divinatoria. Nel corso del tempo il termine «divinazione» ha perso il suo significato originale nel corso dei secoli, rispetto ai tempi antichi e, in particolare, al mondo arcaico greco, in cui ha avuto un enorme valore. La divinazione, infatti, era la forma di conoscenza più genuina, una sorta di porta socchiusa o un ponte che collegava il mondo umano con quello divino. Epidauro era nell'antichità un «paesaggio terapeutico", un complesso architettonico, che comprendeva diversi edifici, a cui la gente veniva da ogni parte della Grecia, per essere curato dal dio Asclepio, una divinità venerata dal 500 prima di Cristo. Epidauro era una struttura molto complessa, una sorta di stato-città o stato sovrano, in grado di ospitare migliaia di adepti.

Figura emblematica e poliedrica, Asclepio viene considerato, ancora oggi, il dio della medicina. A lui è legata una concezione della medicina che è stata definita «medicina templare», di cui Epidauro sarà il centro più importante.

Dobbiamo immaginare inizialmente una certa competizione fra le varie città sedi degli Asklepieia, competizione che venne successivamente accantonata grazie al responso dell'oracolo delfico.

Quest'ultimo riconobbe il ruolo preminente del santuario di Epidauro su tutti gli altri; è Pausania (II, 27, 6) a raccontarci come l'oracolo di Apollo, alla domanda di Apollofane sulla nascita di Asclepio rispose: «O Asclepio, nato a gran gioia di tutti i mortali, che partorì, con me [Apollo] unitasi in amore, la figlia di Flegia, l'amabile Koronis, nella sassosa Epidauro».

L'Asclepieion era situato in una zona pianeggiante e ricca di acque ai piedi del monte Cinorzio; i fedeli giungevano al santuario percorrendo una strada proveniente da Argo o dalla stessa Epidauro. Nella città il culto di Asclepio si impose stabilmente a partire dal VI secolo a. C., raggiungendo la piena egemonia nel IV secolo, vivente lo stesso Ippocrate. Il santuario cinto da mura sorgeva fuori città, collegato a questa da una strada fiancheggiata da statue.

L'ingresso che prendeva il nome di propylon, era decorato con colonne e segnava l'ingresso al santuario; qui le fonti letterarie ricordano la presenza di una grande iscrizione, visibile anche da molto lontano, che esortava i fedeli giunti al santuario: «Puro deve essere chi entra nel tempio odoroso: ma essere puri significa avere pensieri sacri» (Porfirio, De abst., II, 19).

Dopo l'ingresso, una fontana risalente al VI secolo a.C permetteva ai pellegrini di lavarsi e rinfrescarsi dopo il lungo viaggio, oltre ad offrire l'acqua per i riti sacrificali. Il tempio propriamente detto, opera dell'architetto Teodoto, era in pietra calcarea, con la cella al centro contornata da colonne doriche, decorata da marmi policromi e contenente la statua crisoelefantina di Asclepio. 
Asclepio era rappresentato come un uomo maturo, con barba (ma altrove imberbe e giovane), seduto sul trono, con in pugno un bastone e con l'altra mano appoggiata sulla testa di un serpente; accucciato ai suoi piedi, un cane. Cani e serpenti, con le civette oracolari e le capre nutrici, erano animali sacri: la loro lingua era ritenuta capace le leccare, lenire e guarire piaghe. Nei portici antistanti il tempio aveva sede l'abaton, luogo di degenza e dormiveglia, dove i malati passavano le notti immersi nel «sonno incubatorio».

\section{Il rito dell'incubazione}

Il rito dell' «incubatio» è, come vedremo, la pratica di chi dorme per avere dei responsi. Il malato «incubante ad Asclepio" è colui che dorme nell'asclepieo per destarsi dal sonno miracolosamente guarito o, in via subordinata, per ricevere un comandamento o un consiglio sul come comportarsi per guarire.

Nel recinto sacro c'era anche la «thòlos», labirinto circolare dove stava il pozzo sacro, dimora dei sacri serpenti, dove i malati lasciavano le tavolette votive con sopra scritte le loro storie, con i sintomi presentati e i trattamenti ricevuti. Fuori dal recinto completavano l'insieme monumentale le addizioni edilizie, cronologicamente tarde, del ginnasio per gli esercizi fisici, delle terme per i bagni e dell'odéion per le audizioni musicali con finalità terapeutiche.

Il katagòghion, ostello con più stanze ubicate sotto i portici e intorno a cortili, dava ricetto a quanti giungevano in pellegrinaggio al santuario per cercarvi la guarigione. I pellegrini venivano ricevuti da un personale di accoglienza composto da portieri, detentori delle chiavi del tempio, e dagli scribi, che di ciascun pellegrino registravano le offerte e tenevano conto delle condizioni economiche. Il tempio era aperto a tutti, ma ai ricchi erano imposte sovrattasse denominate iatrà. Iatròs era infatti uno dei nomi dati ad Asclepio, che era anche chiamato «re», «eroe», «demone», «despota» e «dio».

Il personale di assistenza era vario: andava dai sorveglianti che vegliavano sopra i dormienti ai pirofori che facevano luce su di essi, agli asclepiadi che sovrintendevano ai sacrifici, ascoltavano i racconti dei sogni, trasmettevano i responsi del dio guaritore. In capo a tutti stava lo ierofante, il supremo "interprete del sacro", la massima sapienza-potenza esistente nel tempio.

Il rituale d'accesso consisteva nei preliminari di purificazione del malato, con lavaggio e breve digiuno, seguiti dal sacrificio della vittima: "un gallo ad Asclepio", come Platone fa dire a Socrate nel Fedone, nell'ultimo discorso agli amici. Sacrificati ad Asclepio erano anche altri animali e chi più aveva, più dava.

Dopo l'offerta, sempre accompagnata dall'invocazione, parte costante in questa ritualità, il malato si distendeva sopra un giaciglio, talora fatto con le pelli stesse degli animali sacrificati: un capro, un bue, una giovenca. Poi dormiva e sognava; talvolta si destava miracolato, altre volte si risvegliava disponibile a confidarsi con l'asclepiade per avere da lui indicazioni terapeutiche. La terapia per la guarigione è interamente basata su un regime che il dio prescrive al paziente, fondandosi su norme dietetiche, esercizi, bagni, "che in parte seguono, in parte precorrono i suoi malori.»

Come si può notare, la terapia non ha niente di miracoloso o divino, ma si basa su una serie di comportamenti che tendono a ripristinare l'equilibrio fisico, alterato dalle varie malattie. L'arte di Asclepio, è, come quella del padre Apollo, arte divina che presuppone un rito di guarigione. Ma nel momento in cui prendiamo in considerazione l'apice di tale sapere, esso contiene già in nuce il germe della sua decadenza. La nascita della cosiddetta medicina tecnica sancirà il progressivo distacco da questa forma di sapere oserei dire iniziatico.

La tecnica - téchne - è infatti sostantivo omerico, è parola greca di derivazione ionica che indica un'arte, un artigianato, un mestiere, storicamente divenuti adulti nell'area geografica dello Ionio e dell'Egeo, tra la Magna Grecia e la madre Grecia e tra questa e l'Asia Minore, nel V secolo a.C.: un'arte in campo medico, legata al nome di Ippocrate. Téchne è un termine multicomprensivo. Comprende il mestiere dell'artigiano, del geometra che misura la terra, del matematico che crea i teoremi, del musico che trova e produce armonie, del medico che cura e ristabilisce la salute degli uomini. E' un mestiere che, in ogni caso, comprende una teoria e una pratica, presenti in diversa misura, ed esige l'applicazione di procedure dell'intelletto.

Nel caso del medico, esercente l'arte della cura con l'intelletto e con la mano, le procedure di questo sapere-potere fanno capo da un lato a forme di conoscenza intellettuale elaborata, dall'altro a pratiche con un consistente contenuto di abilità manuale. Non ultima fra le tecniche, anzi prima dell'importanza del suo oggetto, la medicina è un'attività della ragione umana che si svolge tra l'intellettualità del conoscere e la manualità del fare.

La praxis e il logos verranno a sostituire sempre di più il sapere di Asclepio. Questa visione nella quale il formarsi della medicina detta tecnica è considerato una sorta di filiazione di questa medicina dei templi attraverso una progressiva «desacralizzazione», risale storicamente al filosofo positivista Littré, famoso editore ottocentesco delle opere di Ippocrate.

\section{La nascita della Medicina Ippocratica}

La iatrèia, cioè l'arte di guarire attraverso l'utilizzo di rimedi acquista la sua fisionomia e autonomia mediante una tecnica fattasi matura con Ippocrate.

Ippocrate, il padre indiscusso della moderna medicina, è accreditato da una longevità quasi centenaria che abbraccia oltre metà del V secolo e buona parte del IV. Dal suo primo biografo Sorano, autore di «Genealogia e vita di Ippocrate», il "vecchio di Kos» viene mitizzato come discendente di Asclepio di diciannovesima generazione, a simboleggiare una continuità tra asclepiadi e «tecnici» della salute.

Nonostante questa divina discendenza non possiamo astenerci dal considerare Ippocrate il primo «razionalista» 
ante litteram, demistificatore dell'aura magica che fino al suo avvento aveva ricoperto i rituali di guarigione. A questo proposito sembra opportuno citare le sue stesse parole riguardo all" "antica medicina»:

\begin{abstract}
«La medicina da gran tempo ormai dispone di tutti gli elementi, e il principio e la via sono stati scoperti, grazie ai quali in lungo corso di tempo sono state fatte molte ed egregie scoperte, e il resto nel futuro sarà scoperto, se qualcuno, in grado di farlo e a conoscenza di quanto è già stato scoperto, da questo prendendo le mosse porterà avanti la ricerca. Chi invece, scartato tutto ciò e rifiutandolo, lungo un'altra via e secondo un altro schema s'accinge alla ricerca e asserisce di aver trovato qualcosa, si è ingannato e si inganna, perché è impossibile. E per quali necessarie ragioni sia impossibile, io cercherò di dimostrarlo, dichiarando e dimostrando che cosa è l'arte» (Ippocrate, Opere).
\end{abstract}

Anche Ippocrate che abbiamo definito essere il padre fondatore della medicina tecnica, parla però di arte. All'inizio tra le due figure Ippocrate da una parte ed Asclepio dall'altra ci fu se vogliamo utilizzare un'espressione un po' bizzarra, un tacito accordo, anzi i due continuarono a convivere per molto tempo.

La disquisizione sul mito di Asclepio potrebbe chiudersi qui ma c’è ancora una cosa da ricordare: narra infatti l'evangelista Giovanni $(4,42)$ che Esculapio (la traduzione latina di Asclepio), venerato come un salvatore, fu a lungo un temibile concorrente di Gesù Cristo e il culto di questo dio rivaleggiò per diverso tempo con il Cristianesimo e fu per questo motivo che le strutture legate al culto di Asclepio, furono preda della successiva distruzione cristiana.

\section{Dalla Medicina Occidentale alle Medicine Tradizionali «Altre»}

In ambito terapeutico le ricerche storico-antropologiche registrano in molte culture di tipo sciamanico (Sud America, Siberia, India) un utilizzo molto antico di "piante maestre», ovvero piante che, grazie alle loro proprietà, permettono di raggiungere «Stati non ordinari» di coscienza. Tali stati di coscienza possono sorgere attraverso diverse pratiche, come la meditazione, la respirazione olotropica, lo yoga, la deprivazione sensoriale, o addirittura a livello spontaneo come nel caso dei mistici cristiani.

Dal punto di vista storico, possiamo affermare che in Europa, erano già presenti ai tempi dei greci prima e dell'impero Romano poi, i riti dionisiaci, i misteri di Eleusi, in cui si fa riferimento all'utilizzo di sostanze psicoattive. Elemento tipico del culto di Dioniso è la partecipazione essenzialmente femminile alle cerimonie che si celebravano in diverse zone della Grecia: le Baccanti (chiamate anche Menadi) ne invocavano e cantavano la presenza e, anche per mezzo di maschere (importanti nel culto di Dioniso, che si suppone legato alla nascita della tragedia greca), riproducevano ritualmente il mitico corteo dionisiaco di sileni, satiri e ninfe.

Come ci ricorda McKenna, uno dei più interessanti studiosi del fenomeno sciamanico:

\begin{abstract}
«lo sciamanesimo [...] è una scienza sperimentale che si occupa di un'area di cui non conosciamo nulla. E' importante ricordare che i nostri strumenti epistemologici si sono sviluppati in Occidente in modo assai diseguale. Sappiamo molto su ciò che succede nel cuore dell'atomo, ma assolutamente nulla sulla natura della mente. Non abbiamo alcun indizio. Se la formulazione matematica deve essere il fondamento della certezza ideologica, allora nel regno di ciò che chiamiamo mente non ne abbiamo nessuna. Inconsciamente noi presumiamo ogni genere di cosa, ma, messi alle strette, non siamo in grado di difendere la nostra posizione. Io credo che sia successo questo: a causa della psichedelia da un lato e della fisica quantistica dall'altro, il programma della comprensione razionale della natura è stato spinto tanto oltre da farci raggiungere il cuore irrazionale della natura stessa. Nelle civiltà preippocratiche ciò che oggi definiamo magia, religione e cura erano allora attività appartenenti ad un unico mondo, intese tutte come espressione delle forze sovrannaturali. La malattia e la guarigione, perciò erano considerate frutto di un intervento sovrannaturale» (Op. cit. McKenna, 1991).
\end{abstract}

\section{Le Piante "Maestro" nelle Medicine Tradizionali: dalla foresta al laboratorio}

Oggi, come allora, le ricerche riportano i cambiamenti del comportamento pro sociale in numerosi campioni di persone che hanno fatto uso di diversi tipi di «piante maestre», sia in contesti religiosi, come nel caso dell'ayahuasca per i praticanti della chiesa del Santo Daime, sia in contesti informali.

Le piante contenenti che formano l'ayahuasca sono la Banisteripsis caapi e la Psychotria viridis; quest'ultima contiene dosi elevate di N, N-dimetiltriptamina, conosciuta come DMT, prodotta dal cervello umano e presente in molte sostanze vegetali. I primi studi scientifici sugli effetti psicotropici di questa sostanza iniziarono a essere effettuati a metà degli anni '50 e furono condotti dallo psichiatra e chimico ungherese Stephen Szára, nato nel 1923, che divenne la prima persona a scoprire e riconoscere le proprietà enteogeniche della DMT quando la sperimentò su di sé.

Ma fu Rick Strassmann psichiatra e psicofarmacologo clinico, nato nel 1952, ad avere il merito di accendere l'interesse accademico per la ricerca scientifica delle sostanze psicoattive; Strassman definì la DMT come «la molecola dello spirito", a suggerire le implicazioni profondamente psico-spirituali riguardanti questa sostanza (Bragazzi et al., 2018a-d).

Dopo una serie di ricerche approfondite sugli effetti della somministrazione di DMT, Strassman è arrivato alla conclusione che gli stati alterati di coscienza indotti da 
questa sostanza, presente in molti vegetali sia in Europa sia soprattutto in Sudamerica, potrebbero essere confrontati con le esperienze mistiche:

«Tale sostanza può portarci all'accettazione della coesistenza degli opposti, come la vita e la morte, il bene e il male; una consapevolezza che la coscienza continua dopo la morte; una profonda comprensione dell'unità fondamentale di tutti i fenomeni; e un senso di saggezza o amore che pervade tutta l'esistenza» (Strassman, 2001).

Strassman ha anche osservato che le esperienze di premorte (NDE) possano essere avvicinabili all'esperienza con DMT; infatti molti dei partecipanti ai suoi studi sulla DMT, hanno riferito di contatti con esseri senzienti e disincarnati durante l'esperienza.

Ma quali sono le caratteristiche di questa potente molecola? Secondo Strassman (2001), la DMT può essere considerata la più semplice delle triptamine psicoattive. La sua farmacologia influisce sui siti recettoriali per la serotonina allo stesso modo di altre sostanze come la dietilamide dell'acido lisergico (LSD), la psilocibina e la mescalina. I recettori della serotonina sono diffusi in tutto il corpo umano e possono essere trovati in vari tessuti e organi, inclusi vasi sanguigni, muscoli, ghiandole e pelle.

Nel 1972 lo scienziato vincitore del Nobel Julius Axelrod (1912-2004) riferì di aver isolato la DMT nel tessuto cerebrale umano e altre ricerche hanno dimostrato che la DMT può anche essere trovata nel liquido cerebrospinale che bagna il cervello.

La ghiandola pineale è spesso considerata la fabbrica DMT del corpo umano, e Strassman ha reso popolare la nozione che il cervello rilascia grandi quantità di questo composto durante i sogni e durante la fase di pre-morte. Secondo Strassman, sebbene i tessuti e gli organi come i polmoni, il fegato, il sangue e gli occhi abbiano tutti gli enzimi necessari per convertire la triptamina in DMT, la ghiandola pineale ne è particolarmente ricca; Strassman ha anche ipotizzato che la ghiandola pineale potrebbe continuare a produrre DMT post-mortem per alcune ore.

Recentemente, la dott.ssa Ede Frecska, presidente di Psychiatry presso la Facoltà di Medicina dell'Università di Debrecen, in Ungheria, ha scoperto che la DMT può legarsi al recettore sigma-1 $(\sigma 1 \mathrm{R})$, una proteina trans-membrana che può essere comunemente trovata in tutto il corpo e, soprattutto, in alcune regioni del sistema nervoso centrale. Questo recettore svolge un ruolo chiave nel proteggere le cellule dall'apoptosi quando la concentrazione di ossigeno è criticamente bassa (in altre parole, in condizioni di ipossia), lasciando spazio all'affascinante argomento secondo cui la DMT può effettivamente essere prodotta e rilasciata in grandi quantità durante la morte in un ultimo tentativo di mantenere vive le cellule umane. Tuttavia, dobbiamo ancora scoprire che DMT è effettivamente prodotto nella ghiandola pineale degli esseri umani.

Lasciando da parte per un po' la DMT, possiamo dire che la ghiandola pineale è considerata centrale in diverse pratiche religiose e mistiche in tutto il mondo, e ha svolto un ruolo importante nelle teorie di Galeno (circa 130-210 CE) e René Descartes (1596-1650) (López-Muñoz \& Alamo, 2011).

Recentemente, Roney-Dougal (1989; 1991; 2001) ha suggerito che la ghiandola pineale e la sua neurochimica e neuro-anatomia possano essere di cruciale importanza nel verificarsi dei cosiddetti «fenomeni psi» (Rogo, 1975; Rogo, 1976) e indica l'associazione fatta dagli yogi tra la ghiandola pineale e l'ajna chakra, il centro psichico yogico che controlla le esperienze psi in quelli con la kundalini risvegliata (Luke, 2012; Miller, 1978; Satyananda, 1972).

Nello studio dell'attività della ghiandola pineale tra un metodo scientifico corretto e rigoroso e un atteggiamento mistico e teologico, il lavoro del medico italiano Paolo Lissoni, oncologo e pioniere nell'approccio alla medicina psico-neuro-endocrino-immunologica (PNEI) è davvero prezioso e fondamentale (Lissoni, 1999).

Secondo un recente lavoro (Lissoni et al., 2001) uno dei principali progressi nella conoscenza della mediazione neuro-chimica della vita psico-spirituale è stata la scoperta del ruolo fondamentale della ghiandola pineale nella regolazione del sistema immunitario. Nella visione mistica di Lissoni dell'Uomo come immagine del Dio trino nella sua natura biochimica, psicologica e spirituale, la ghiandola pineale può essere considerata il ponte fisico/ neuro-anatomico tra questi aspetti multidimensionali (Lissoni, 1999; Lissoni et al., 2001).

Andando oltre con la sua visione fortemente spirituale del processo di guarigione, Lissoni ha scoperto che i diversi neurormoni prodotti dalla ghiandola pineale potrebbero aiutare i pazienti oncologici a ripristinare la naturale resistenza immuno-biologica contro la crescita del tumore, attraverso la regolarizzazione del ritmo di produzione di quattro indoli pineali principali prodotti e rilasciati in specifici intervalli temporali: i) 5-metossitriptolo (5-MLT) a mezzogiorno, ii) 5-metossitriptamina (5-MT) al tramonto, iii) melatonina (MLT) durante il notte e iv) acido acetico 5-metossi-3-indolo (5-MIAA) al mattino presto.

Questa regolarizzazione finemente sintonizzata è in grado di aiutare il paziente a riconnettersi alla sua dimensione spirituale per guarire dalla sua malattia. Nelle parole di Lissoni (1999), la gerarchia apicale della ghiandola pineale nella psicobiologia umana è provata dal fatto che rappresenta l'unica struttura del corpo umano in grado di trasdurre la luce e il magnetismo dell'universo in una risposta biologica, modulando la funzionalità dell'organismo in piena armonia con l'ambiente circostante (Lissoni, 1999).

\section{Futuri sviluppi e prospettive delle terapie con sostanze psicoattive}

Sono necessarie ulteriori ricerche su questa direzione per comprendere meglio le proprietà delle triptamine come la DMT e i loro effetti biofisici e le implicazioni sulla percezione umana e sulla comunicazione cerebrale.

Per concludere, l'esperienza contemporanea riportata 
dai ricercatori ci sta offendo dati molto incoraggianti sui benefici di questo tipo di esperienze. La ricerca sviluppata negli USA e in Europa in Spagna e Svizzera, tende a seguire protocolli di ricerca, in cui giocano un ruolo fondamentale il Set (lo stato attuale ma anche la storia clinica e biografica del paziente), il Setting (l'ambiente in cui avviene l'esperienza) e la sostanza (tipologia, dosaggio, qualità e quantità).

Il Setting solitamente prevede una stanza accogliente con un divano che verrà utilizzato durante la sessione, una maschera per coprire gli stimoli visivi, delle cuffie collegate ad uno stereo e operatori, pscioterapeuti e medici opportunamente formati, che hanno il compito di accompagnare la persona attraverso l'esperienza di cura attraverso l'utilizzo della sostanza.

In particolare in California è in corso una sperimentazione con l'utilizzo di MDMA, per pazienti con una diagnosi di PTSD (disturbo post traumatico da stress). Nell'ambito della depressione maggiore e dell'ansia legata al fine vita, si stanno valutando i benefici dell'utilizzo della Psilocibina, una sostanza contenuta nei funghi utilizzati da millenni nell'antica tradizione Huichol messicana. Lo schema della ricerca prevede la valutazione dei sintomi pre e post l'utilizzo della sostanza e prevede la somministrazione di questionari e l'utilizzo di sessioni di psicoterapia per integrare le esperienze vissute e trovare collegamenti utili alla persona nel proseguio della propria vita quotidiana. I pazienti spesso riportano esperienze di dissoluzione dell'Ego che, grazie alla tecniche di neuroimaging, sappiamo essere connesse con una netta diminuzione di attività di una zona del cervello chiamata DMN, Default Mode Network; tale zona sembra essere iperattiva durante episodi di «ruminazione e rimuginio", tipiche negli stati depressivi, e gioca un ruolo importante anche nella capacità di introspezione e nello sviluppo della capacità di saper «leggere» e interpretare le emozioni degli altri.

Nell'ambito delle dipendenze sia l'utilizzo di sostanze derivate da piante come nel caso dell'Ayahuasca sia l'utilizzo di sostanze chimiche come ad esempio l'MDMA, sono in grado di far emergere di memorie antiche precedentemente rimosse, facilitando l'elaborazione dei traumi che possono esserne la base.

I campioni statistici sono ancora limitati, per ovvie ragioni «pratiche» e per la difficoltà ad ottenere i permessi, ma i risultati hanno messo in luce le grandi proprietà di questi antichi rimedi, in un contesto sicuro e controllato.

Le sfide per il futuro sull'utilizzo terapeutico delle sostanze psicoattive sono ancora molte, una possibilità concreta potrebbe essere quella di rivitalizzare l'utilizzo rituale delle sostanze unendo passato e presente, antiche conoscenze e moderna scienza a servizio della cura.

\section{Bibliografia}

Ahearne-Kroll, S.P. (2014). The afterlife of a dream and the ritual system of the Epidaurian Asklepieion. Archiv fur Religionsgeschichte, 15(1), 35-51.

Al-Balhan, E. M., Khabbache, H., Watfa, A., Re, T.S., Zerbetto, R. \& Bragazzi, N.L. (Hrsg.). (2018). Psychometric Evaluation of the arabic version of the nomophobia questionnaire: confirmatory and exploratory factor analysis - implication from a pilot study in Kuwait among university students. Psychology Research and Behavioural Management, 11, 471-482.

Aldridge, D. (2000). Spirituality, Healing and Medicine: Return to the Silence. London: Jessica Kingsley.

Bragazzi, N.L., Khabbache, H., Perduca, M., Neri, B., Firenzuoli, F., Penazzi, G., Simões, M., Zerbetto, R. \& Re, T.S. (2018a). Para-Psychology, N,N-Dimethyltryptamine and the Pineal Gland. Cosmos and History: The Journal of Natural and Social Philosophy, 12(2), 228-238.

Bragazzi, N.L., Khabbache, H., Vecchio, I., Martini, M., Perduca, M., Zerbetto, R. \& Re, T.S. (2018b). Ancient Shamanism and Modern Psychotherapy: From Anthropology to Evidence-Based Psychedelic Medicine. Cosmos and History: The Journal of Natural and Social Philosophy, 14(1), 142-152.

Bragazzi, N.L., Khabbache, H., Vecchio, I., Martini, M., Zerbetto, R. \& Re, T.S. (2018c). Neurotheology of Islam and higher consciousness states. Cosmos and History: The Journal of Natural and Social Philosophy, 14(2), 315-321.

Bragazzi, N.L., Re, T.S., Zerbetto, R., Firenzuoli, F. \& Gensini, G. F. (2018d). Umbrella review of medicinal cannabis. Phytomedicine Journal, 2018 (submitted).

Bulkeley, K. (2008). Dreaming in the World's Religions: A Comparative History. New York: University Press.

Drury, N. (2011). Stealing Fire from Heaven: The Rise of Modern Western Magic. Oxford: University Press.

Edelstein, E. J. \& Edelstein, L. (1945). Asclepius. Collection and Interpretation of the Testimonies. Baltimore: John Hopkins Press.

Firenzuoli, F., Maggini, V., Re, T.S., Sivelli, F., Gallo, E. \& Gensini, G.F. (2018). Clinical phytotherapy and herbal pharmacovigilance [eBook; www.sci9.org/ebooks].

Firenzuoli, F., Sivelli, F., Maggini, V., Re, T.S., Gallo, E. \& Gensini, G. F. (2018). Medicinal Cannabis in Italy: Current Events and Perspectives [eBook; www.sci9.org/ebooks].

Gesler, W.M. (1993). Therapeutic Landscapes: Theory and a Case Study of Epidauros, Greece. Environment and Planning D: Society and Space,11(2), 171-189.

Gesler, W.M. (2003). Healing Places. Rowman \& Littlefield.

Gulluni, N., Re, T.S., Loiacono, I., Lanzo, G., Gori, L., Macchi, C., Epifani, F., Bragazzi, N. L. \& Firenzuoli, F. (2018). Cannabis Essential Oil: A Preliminary Study for the Evaluation of the Brain Effects. Evidence-Based Complementary and Alternative Medicine, 2018. https://doi.org/10.1155/2018/1709182

Hart, G. D. (2000). Asclepius: The God of Medicine. London: Royal Society of Medicine Press.

Hoot, D. R. (2014). The Sanctuary of Asclepius at Epidaurus in Roman Times. Gainesville: University Press of Florida.

Lissoni, P. (1999). The pineal gland as a central regulator of cytokine network. Neuro Endocrinol Letters, 20(6), 343-349.

Lissoni, P., Cangemi, P., Pirato, D., Roselli, M.G., Rovelli, F., Brivio, F., Malugani, F., Maestroni, G. J., Conti, A., Laudon, M., Malysheva, O. \& Giani, L. (2001). A review on cancer-psychospiritual status interactions. Neuro Endocrinology Letters, 22(3), 175-80.

López-Muñoz, F. \& Alamo, C. (2011). Cartesian theories on the passions, the pineal gland and the pathogenesis of affective disorders: an early forerunner. Psychological Medicine 41(3), 449-51.

Luke, D. (2011). Discarnate entities and dimethyltryptamine (DMT): Psychopharmacology, phenomenology and ontology. Journal of the Society for Psychical Research, 75, 26-42. 
Luke, D. (2012). Psychoactive substances and paranormal phenomena: A comprehensive review. International Journal of Transper sonal Studies, 31(1), 97-156.

Malatesta, M. (Hrsg.). (2015). Doctors and Patients: History, Representation, Communication from Antiquity to the Present. San Francisco: University of California Medical Humanities Press.

McKenna, T. (1992). Food of the Gods. New York: Bantam.

Meier, C.A. (2012). Healing Dream and Ritual: Ancient Incubation and Modern Psychotherapy. Einsiedeln: Daimon.

Perriam, G. (2015). Sacred spaces, healing places: therapeutic landscapes of spiritual significance. Journal of Medical Humanities, 36(1), 19-33.

Petsalis-Diomidis, A. (2010). Truly Beyond Wonders: Aelius Aristides and the Cult of Asklepios. Oxford: University Press.

Pettis, J.B. (2015). The Sleeper's Dream: Asclepius Ritual and Early Christian Discourse. Piscataway: Gorgias Press.

Re, T.S., Palma, J., Martins, J.E. \& Simões, M. (2016). Transcultural perspective on consciousness: Traditional use of ayahuasca in psychotherapy in the 21st Century in Western World. Cosmos and History: The Journal of Natural and Social Philosophy, 12(2), 237-249.

Re, T.S. \& Ventura, C. (2015). Transcultural Perspective on Consciousness: a bridge between Anthropology, Medicine and Physics. Cosmos and History: The Journal of Natural and Social Philosophy, 11(2), 228-241.

Renberg, G. H. (2017). Where Dreams May Come Incubation Sanctuaries in the Greco-Roman World (Vol. I). Leiden and Boston: Brill.

Roney-Dougal, S.M. (1989). Recent findings relating to the possible role of the pineal gland in affecting psychic ability. Journal of the Society for Psychical Research, 55, 313-328.
Roney-Dougal, S. M. (1991). Where Science and Magic Meet. Shaftesbury, Dorset: Element Books.

Roney-Dougal, S. M. (2001). Walking between the worlds: Links between Psi, Psychedelics, Shamanism and Psychosis. Glastonbury: Psi Research Centre.

Walton, A. (1894). The cult of Asklepios. Boston: Ginn \& Company. Williams, A. (1998). Therapeutic Landscapes in Holistic Medicine. Social Science and Medicine. 46(9), 1193-1203.

Winkelman, M.J. (1992). Shamans, Priests and Witches. A Cross-Cultural Study of Magico-Religious Practitioners. Tempe: Arizona State University.

\section{L'autrice}

Tania Re, Laurea in Psicologia Clinica e di Comunità, è Antropologa specializzata in Antropologia della Salute ed Etnomedicina; è Terapista Complementare e Psicologa in Svizzera. Socia Fondatrice della Cattedra Unesco «Salute Antropologia, Biosfera e sistemi di cura» presso l'Università di Genova (IT), è ricercatrice presso il Centro Regionale Toscana per la Fitoterapia (CERFIT) - Ospedale Careggi, Firenze (IT). Da sempre è interessata a comprendere i sistemi provenienti da antiche tradizioni di cura che connettono mente, corpo e spirito.

\section{Contatto}

E-Mail: tania.re77@gmail.com 